\title{
Renal safety of tenofovir disoproxil fumarate and entecavir in liver transplant patients: a nationwide Korean registry study
}

\author{
Juhan Lee ${ }^{1}$, Shin Hwang ${ }^{2}$, Kwang-Woong Lee ${ }^{3}$, Jong Man Kim ${ }^{4}$ Je Ho Ryu ${ }^{5}$, Bong-Wan Kim ${ }^{6}$ Dong Lak Choi ${ }^{7}$, \\ Young Kyoung You ${ }^{8}$, Dong-Sik Kim ${ }^{9}$, Myoung Soo Kim ${ }^{1}$ \\ ${ }^{1}$ Division of Transplantation, Department of Surgery, Severance Hospital, Seoul, Korea \\ ${ }^{2}$ Division of Hepatobiliary, Department of Surgery, Asan Medical Center, University of Ulsan College of Medicine, Seoul, Korea \\ ${ }^{3}$ Division of Hepatobiliary, Department of Surgery, Seoul National University Hospital, Seoul, Korea \\ ${ }^{4}$ Division of Transplantation, Department of Surgery, Samsung Medical Center, Seoul, Korea \\ ${ }^{5}$ Division of Hepatobiliary, Department of Surgery, Pusan National University Yangsan Hospital, Yangsan, Korea \\ ${ }^{6}$ Division of Hepatobiliary, Department of Surgery, Ajou University School of Medicine, Suwon, Korea \\ ${ }^{7}$ Division of Hepatobiliary, Department of Surgery, Daegu Catholic University Medical Center, Daegu, Korea \\ ${ }^{8}$ Division of Hepatobiliary, Department of Surgery, Seoul St. Mary's Hospital, College of Medicine, The Catholic University of Korea, Seoul, Korea \\ ${ }^{9}$ Division of Hepatobiliary, Department of Surgery, Korea University College of Medicine, Seoul, Korea
}

Background: Entecavir and tenofovir are currently the first-line drugs in liver transplantation (LT) patients for the prevention of hepatitis B virus recurrence. Despite its proven efficacy, the renal safety of tenofovir in LT patients has not been well defined. We aimed to assess the impact of tenofovir on renal function compared to that of entecavir after LT.

Methods: We analyzed 817 LT patients treated with entecavir $(n=366)$ or tenofovir $(n=451)$ between 2014 and 2017. The estimated glomerular filtration rate (eGFR) was calculated using the Chronic Kidney Disease Epidemiology Collaboration equation. Renal function deterioration was defined as progression of chronic kidney disease stage and $\geq 20 \%$ eGFR decline from 1 month after LT.

Results: Mean eGFR at 1 month after LT was $91.1 \pm 22.0 \mathrm{~mL} / \mathrm{min} / 1.73 \mathrm{~m}^{2}$ for entecavir and $89.1 \pm 23.7 \mathrm{~mL} / \mathrm{min} / 1.73 \mathrm{~m}^{2}$ for tenofovir group $(P=0.312)$. During a median follow-up of 29 months, the cumulative incidence of renal function deterioration was significantly higher in the tenofovir group than in the entecavir group ( $355.5 \%$ vs. $27.1 \%, P=0.01)$. Tenofovir (odd ratio [OR], $1.52 ; 95 \%$ confidence interval [Cl], 1.11-2.08; $P=0.009)$, old age $(\mathrm{OR}, 1.45 ; 95 \% \mathrm{Cl}, 1.01-2.09 ; \mathrm{P}=0.044)$, low body mass index $(\mathrm{OR}, 2.02 ; 95 \%$ $\mathrm{Cl}, 1.44-2.84 ; \mathrm{P}<0.001)$, and diabetes mellitus $(\mathrm{OR}, 1.68 ; 95 \% \mathrm{Cl}, 1.18-2.08 ; \mathrm{P}=0.009)$ were independent risk factors for renal function deterioration.

Conclusions: The use of tenofovir was significantly associated with renal function deterioration in LT patients. In the setting of $\mathrm{LT}$, tenofovir should be used with caution in high-risk patients and renal function should be carefully monitored.

Corresponding author: Juhan Lee

E-mail: juhan1108@gmail.com

(C) The Korean Society for Transplantation

This is an Open Access article distributed under the terms of the Creative Commons Attribution Non-Commercial License (http://creativecommons.org/licenses/by-nc/4.0/) which permits unrestricted non-commercial use, distribution, and reproduction in any medium, provided the original work is properly cited. 